\title{
In Situ HREM of Crystallization Reactions
}

\author{
R. Sinclair and K.-H. Min \\ Department of Materials Science and Engineering, Stanford University, Stanford, CA 94305-2205
}

In-situ high-resolution electron microscopy (HREM) is ideally suited for the investigation of solid-state crystallization behavior. The well-known image contrast of an amorphous material is quite clearly, and often dramatically, replaced by that of the crystalline phase. However compared to other techniques, the observations are now being made at the atomic or nano-scale level so the detail provided is quite different in scope. This article reviews work in this field.

Parker was the first researcher to utilize a heating holder to achieve controlled elevated temperatures while maintaining highresolution imaging conditions (e.g. [1]). His work showed that kinetic data for the solid-phase epitaxial regrowth of silicon were the same by in situ HREM as those obtained by bulk Rutherford backscattering and high-voltage electron microscopy studies [2]. Morgiel et al. (e.g. [3]) extended this to the direct crystallization of amorphous silicon deposited on oxidized silicon substrates. Of particular note was the existence of pre-existing crystal embryos, the majority of which did not form viable nuclei. In both cases, there was no clear atomic ledge mechanism for the silicon crystal growth, even though this is an often-accepted mechanism [4]. Furthermore the growth often occurred in "spurts" with many atoms assuming the crystalline structure between individual video frames, an observation that was confirmed by Guillemet et al. in through-foil HREM investigation [5].

Konno utilized the in situ method combined with differential scanning calorimetry to study the metal-mediated crystallization of amorphous silicon and germanium [6-8]. In situ HREM clearly showed that the much-reduced crystallization temperatures arose from crystal nucleation within the metal, followed by more rapid substitutional diffusion of Si or Ge atoms through the metallic matrix than can occur at the amorphous-crystalline interface. This mechanism was also realized in the silicide-mediated crystallization of silicon, as described by Hayzelden and Batstone [9]. Kinetic and in situ studies demonstrated that an equivalent mechanism occurs during the crystallization of amorphous co-sputtered Al-Si alloys [10]. Progression of this work to carbon crystallization is described elsewhere [11]. A ledge mechanism for the Ag-Si system is also very clear when the metal-semiconductor interface is parallel to $\{111\}$ type planes [7].

Room temperature thin film deposition of oxides also often results in an amorphous material. Because the melting points are generally higher than those of other materials (and so lower diffusion rates), crystallization may require higher annealing temperatures, which makes the in situ experiment more difficult. However, Fig. 1 shows in situ HREM taken during the crystallization of amorphous $\mathrm{Ta}_{2} \mathrm{O}_{5}$, a candidate material for high dielectric constant applications in integrated circuits. Pictures in this sequence show that the growth of the (001) planes take place via a ledge mechanism though it is not a perfect single plane one. The growth activation energy was found to be $4.8 \mathrm{eV}$, comparable to that found for $\mathrm{Ta}_{2} \mathrm{O}_{5}$ powder particle coarsening $(5.6 \mathrm{eV})[12]$.

In summary, crystallization mechanisms are clearly elucidated by in situ HREM studies. One drawback is that because the atomic 
positions are not evident in the amorphous material, the exact attachments are not always clear, although a ledge mechanism normally is very distinctive when it is present.

\section{References}

[1] R. Sinclair and M.A. Parker, Nature 322 (1986) 531.

[2] M.A. Parker and R. Sinclair, Proc. ICEM-XI 2 (1986) 991.

[3] R. Sinclair et al., Ultramicroscopy 51 (1993) 41.

[4] R.M. Drosd and J. Washburn, J. Appl. Phys. 53 (1982) 397.

[5] J.P. Guillemet et al., Proc. ICEM-XIII (1994) 449.

[6] T.J. Konno and R. Sinclair, Philos. Mag. B, 66 (1992) 749.

[7] T.J. Konno and R. Sinclair, Philos. Mag. B, 71 (1995) 163.

[8] T.J. Konno and R. Sinclair, Philos. Mag. B, 71 (1995) 179.

[9] C. Hayzelden and J.L. Batstone, J. Appl. Phys. 73 (1993) 8279.

[10] T.J. Konno and R. Sinclair, Mater. Chem. Phys. 35 (1993) 99.

[11] R. Sinclair et al., Proc. ICEM-XV (2002) in press.

[12] J.M. Heints et al., Ceram. Int. 18 (1992) 263.
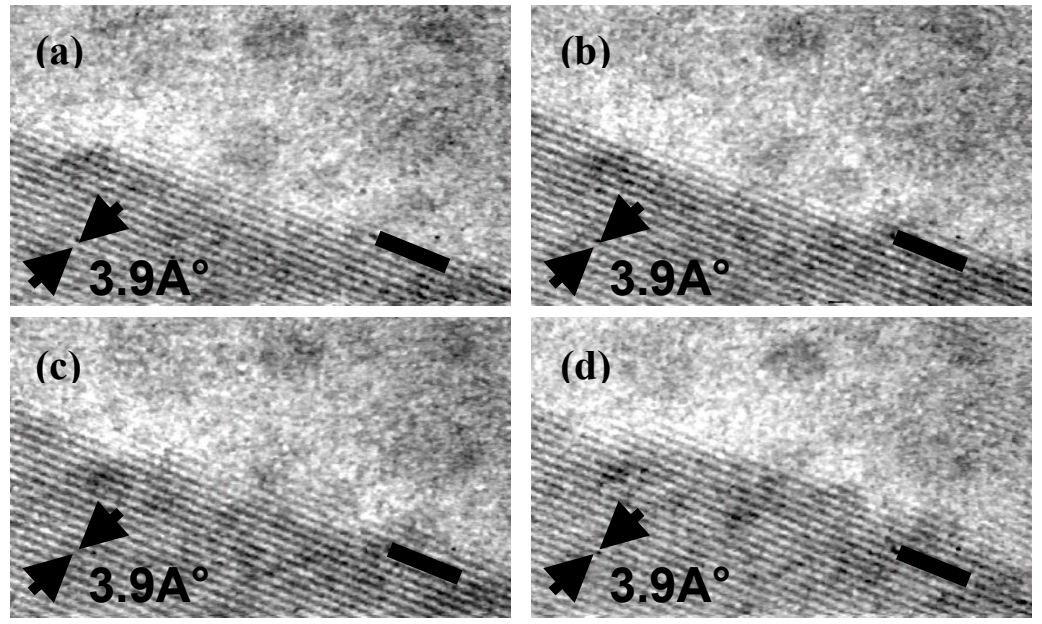

FIG. 1 A sequence of HREM images reproduced from videotape recorded during in-situ heating of $\mathrm{Ta}_{2} \mathrm{O}_{5}$ film with an interval of 2 seconds. 